\title{
A bedside computer in foetal assessment
}

\author{
JAMES T. CURRAN \\ Bio-Engineering Unit, University of Strathclyde, Glasgow
}

\begin{abstract}
Summary
The time has now come when mere survival of an infant can no longer be considered the index of successful obstetrics. It is thought that as many as one in ten surviving infants suffer some degree of damage in the process of labour. The two techniques to be described in this paper are attempts to derive methods for prognosing the ability of a foetus to withstand this process.
\end{abstract}

1. The Foetal Electrocardiogram: The examination of the FECG by the traditional methods of cardiology have proved very disappointing and unrewarding. If, however, the FECG is merely used as a means of recording the beat-to-beat interval of the foetal heart, statistical evaluation of this information can yield a valuable prognostic index.

2. The Foetal Ballistocardiogram: The purpose of the foetal heart (or for that matter the adult heart) is not to generate electrical signals but to propel blood through the circulation. One method of measuring this function in the adult is the ballistocardiogram and the application of modern signal recovery techniques has made it possible to derive the foetal ballistocardiogram.

RECENT YEARS have seen such an impressivereduction in both maternal and foetal mortality in childbirth that more attention must be turned to the question of infant morbidity. Although some $3 \%$ of all births in the U.K. result in death of the child (British Perinatal Survey, 1968) at least the same percentage of infants suffer demonstrable mental damage and as many as $10 \%$ may be slightly affected. In absolute terms this represents 28,000 mentally handicapped children per annum, half of which require special schooling at a cost of $£ 30,000,000$ to the nation. By far the greatest part of this mortality and morbidity is due to hypoxia occurring before and during birth. If the infants at risk could be accurately identified, operative measures to expedite delivery could probably lead to a considerable improvement in the situation.

Clearly there is great need to gather more information on the unborn foetus. Due to its inaccessibility the traditional methods of medical examinations are inapplicable and the fruits of modern technology must be brought to bear. This paper will present two such methods - the foetal electrocardiograph and the foetal ballistocardiograph.

\section{The foetal electrocardiograph (FECG)}

Although the FECG was described by Cremer as long ago as 1906 efforts to interpret the signal by the standards of adult cardiology have been most unrewarding. The advent of the small portable computer has provided a fresh impetus and promises more encouraging results.

Briefly the FECG is obtained from the maternal abdomen by a two-channel cancellation system previously described (Curran, Evans \& MacGregor, 1969) in which the maternal ECG is suppressed leaving the FECG as the larger waveform. This waveform is then passed to a small computer (Nuclear Chicago 7100) which can operate in two modes.

(a) Transient average. In this mode of operation the computer sums successive cycles of the input waveform only over the interval in which the foetal heart is beating thereby recovering all the detail of the FECG despite the other interference present. Fig. 1 is an example of such recovery. (A detailed

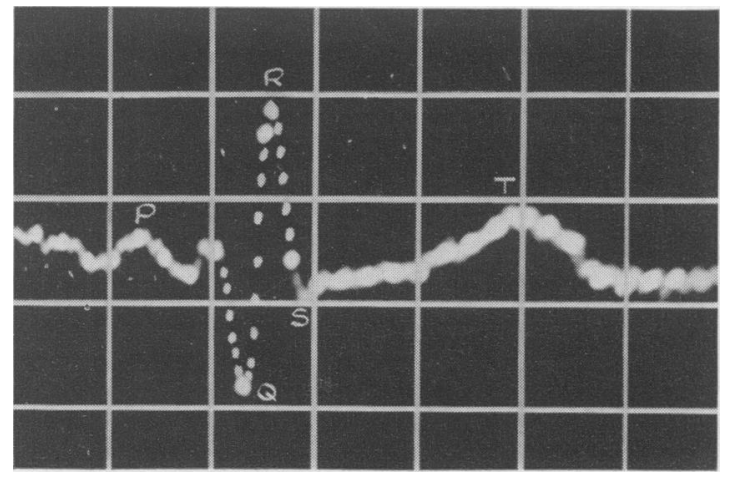

Fig. 1. A detailed FECG complex recovered by transient averaging. 
description of the process has been given by Hon \& Lee (1963).)

(b) Interval histogram. In this mode the computer constructs a frequency histogram of the intervals between foetal heart beats over about 3000 cycles. Fig. 2 gives examples of two such histograms with the $\mathrm{X}$-axis calibrated as beats/min for convenience.
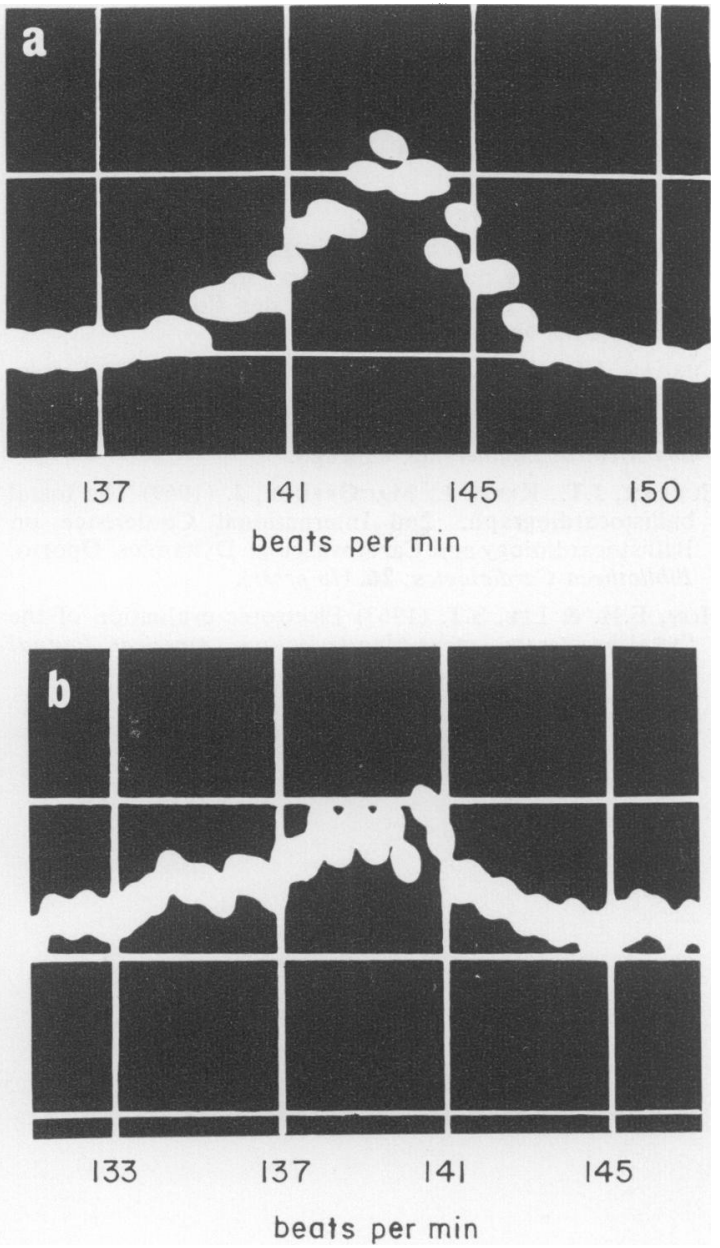

FIG. 2. Two examples of interval histograms.

The impression given by consideration of these histograms is that type (a) is associated with a healthy foetus and type (b) with an unhealthy foetus. Standard statistical methods have been applied to 100 histograms obtained from near term pregnancies to yield four indices:

(i) The range (max-min)

(ii) The mean (millisecs).

(iii) The standard deviation (millisecs).

(iv) The coefficient of variation (SD as percentage of mean).
All four indices have been compared with the results of labour as judged by the infants Apgar score at birth (Apgar, 1953). The range is in no way prognostic, both the mean and the standard deviation are prognostic but the coefficient of variation proves to be highly significantly prognostic $(P<0.005)$.

Clearly any measure of foetal heart activity can be used to construct a histogram and both ultrasonic doppler techniques and phonocardiography have been demonstrated as suitable. However, the FECG remains attractive as it is utterly harmless to mother and child and provides a waveform of ideal electric form for further processing.

The foetal ballistocardiograph (FBCG)

A little sober reflection yields the obvious conclusion that the function of the heart is not to produce electrical signals but to propel blood around the
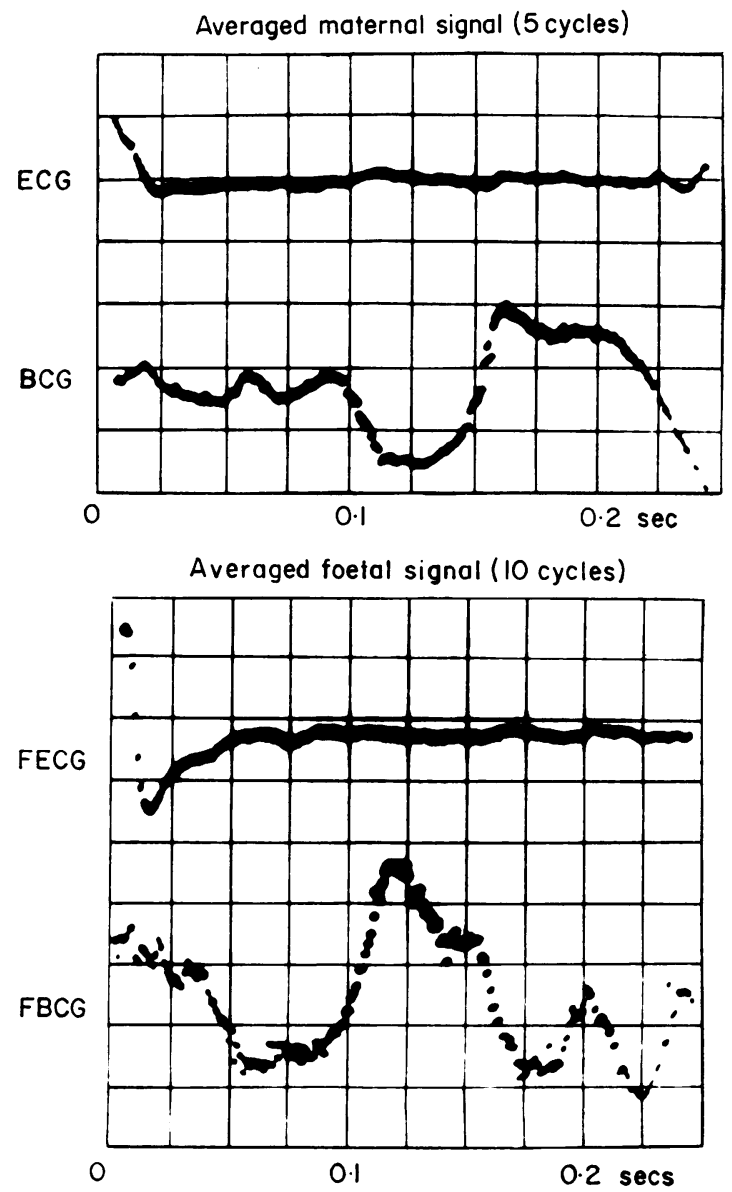

FIG. 3. Maternal and foetal ECG and BCG recovered from the same section of recording. 
body. One measure of this function is the ballistocardiograph. This method, which has never enjoyed popularity in Britain, is worthy of more serious consideration when modern engineering technology is applied to it.

An air bearing BCG table with a novel, very sensitive, transducer was built by the National Engineering Laboratories, East Kilbride (Curran, Kerr \& MacGregor, 1969) to examine the possibility of recovering the FBCG. The output of the transducer contains the disturbances due to both the maternal and foetal heart beats but, with the FECG already available to instruct the computer to operate, in its transient average mode, on the combined waveform, the FBCG can be easily demonstrated. Fig. 3 shows the signals recovered from the same recording, firstly averaging the mother's BCG and secondly averaging the FBCG. The FBCG has the same detailed waveform as that of the adult though naturally it occurs over a shorter time-interval.

The clinical usefulness of the technique has not yet been established but it does present, for the first time, the possibility of obtaining information on mass-flow relationships within the foetus by a noninvasive technique.

No one or two techniques in obstetrics can be viewed in isolation without regard to the complex interaction of maternal and foetal factors. The consideration of labour under the classical headings of the powers, the passages and the passenger still holds good and while the mere survival of the infant was a considerable achievement the emphasis was on the first two. Now that the quality of life has been recognized as equally important much more emphasis must be placed upon monitoring the potential passenger not only during his journey but before it commences.

\section{References}

APgar, V. (1953) A proposal for a new method of evaluation of the newborn infant. Anaesthetics and Analgesics, 32, 260.

British Perinatal Survey (1958).

Cremer, M. (1906). Ueber die direkte Ableitung der Akitionsstrome des menschuchen Herzens vom Oesophagus und uber das Elektrokardiogram des Fotus. Münchener Medizinische Wochenschrift, 53, 811.

Curran, J.T., Evans, J.H. \& MacGregor, J. (1969) A practical index of foetal viability by on-line analysis of cardiac cycle interval. Eighth International Conference on Biological and Medical Engineering, Chicago.

Curran, J.T., Kerr, J., MacGregor, J. (1969) The foetal ballistocardiograph. 2nd International Conference on Ballistocardiology and Cardiovascular Dynamics, Oporto. Bibliotheca Cardiologica, 26. (In press).

HoN, E.H. \& LeE, S.T. (1963) Electronic evaluation of the foetal heart rate-measuring technique. American Journal of Obstetrics and Gynecology, 84, 6. 